\title{
Desove y desarrollo intracapsular del caracol marino Hexaplex nigritus (Neogastropoda: Muricidae) en laboratorio
}

\author{
Andrés M. Góngora-Gómez ${ }^{1}$, Misael Pinzón-Zúñiga ${ }^{2}$, Juan A. Hernández-Sepúlveda ${ }^{1}$, \\ Manuel II García-Ulloa ${ }^{3}$, Brenda P. Villanueva-Fonseca ${ }^{2} \&$ Manuel García-Ulloa ${ }^{1 *}$ \\ 1. Instituto Politécnico Nacional, Centro Interdisciplinario de Investigación para el Desarrollo Integral Regional, Unidad \\ Sinaloa, Departamento de Acuacultura, Guasave, Sinaloa, México; gogam69@hotmail.com, juanhese@hotmail.com, \\ turbotuag@hotmail.com \\ 2. Universidad Autónoma de Occidente, Unidad Regional Guasave, Sinaloa, México; Misael_pz@outlook.com, brenda- \\ paulina1984@hotmail.com \\ 3. Instituto de Ecología, Universidad Nacional Autónoma de México, Ciudad de México, México; \\ manuel.gug@hotmail.com \\ * Correspondencia
}

Recibido 11-VI-2020. Corregido 12-VIII-2020. Aceptado 18-VIII-2020.

\begin{abstract}
Spawning and intracapsular development of the marine snail Hexaplex nigritus (Neograstropoda: Muricidae) in the laboratory. Introduction: The marine snail Hexaplex nigritus is a heavily exploited muricid in the Gulf of California for consumption and handcrafts. When they reproduce, adults aggregated in the form of artificial reefs facilitating their identification and extraction, situation that has reduced their population in that area. Objective: In order to investigate the spawning and intracapsular and larval development of this species as tools for its production in captivity for repopulation purposes. Methods: Eighteen brooders were collected and kept in a closed system with controlled parameters and feeding from April 2017 to September 2018. Growth of parents, spawning of ovigerous masses, number of capsules, and number of embryos and larvae within the capsules, were registered. The main morphological structures of embryos and larvae were documented according to their development. Results: Intracapsular embryonic development and veliger larva until its establishment lasted 14-15 and 31-32 days, respectively. Each 80-88 mm length female produced around 4 ovigerous masses, 627 capsules and 663993 extracapsular veliger larvae per year. Conclusions: Easy adaptation and reproductive potential shown by $H$. nigritus in the laboratory are presented as promising strategies for its repopulation and conservation.
\end{abstract}

Key words: conservation; controlled conditions; embryonic development; gastropods; Gulf of California; reproductive strategy.

Góngora-Gómez, A.M., Pinzón-Zúñiga, M., Hernández-Sepúlveda, J.A., García-Ulloa, M.II, Villanueva-Fonseca, B.P., \& García-Ulloa, M. (2020). Desove y desarrollo intracapsular del caracol marino Hexaplex nigritus (Neogastropoda: Muricidae) en laboratorio. Revista de Biología Tropical, 68(4), 1143-1158.

El caracol chino negro Hexaplex (= Muricanthus) nigritus es un gastrópodo marino endémico del Golfo de California (Poutiers, 1995), cuyo hábitat en el área infralitoral presenta un rango de profundidad que abarca desde pocos centímetros hasta 60 metros, en sustratos de arena, lodo y grava (Stump, 1975;
Brusca, Kimrey, \& Moore, 2004); puede 1legar a medir hasta $143.5 \mathrm{~mm}$ de longitud total (Cudney-Bueno \& Rowell, 2008). A pesar de su importancia comercial, existe poca información de su desove y desarrollo intracapsular.

Como parte de su estrategia reproductiva, Hexaplex nigritus desova masivamente 
produciendo cápsulas en donde ocurre el desarrollo embrionario hasta un estado de larva velígera (Cudney-Bueno, Prescott, \& Hinojosa-Huerta, 2008), en aglomeraciones de hasta miles de individuos en zonas de baja profundidad que semejan arrecifes artificiales que sirven de hábitat para decenas de especies de invertebrados en su estado juvenil, especialmente filtradores (Prescott \& Cudney-Bueno, 2008; Góngora-Gómez, García-Ulloa, Domínguez-Orozco, \& Camacho-Sánchez, 2011). Sin embargo, esta característica reproductiva de forma gregaria facilita a los pescadores su identificación y extracción, lo que ha reducido drásticamente su población en las zonas donde principalmente se distribuye (noreste y sureste) dentro del Golfo de California (Cudney-Bueno et al., 2008; Góngora-Gómez et al., 2011).

Hexaplex nigritus se captura en la zona intermareal a mano o mediante buceo autónomo; también, es atrapado de manera incidental en redes de fondo representando un recurso secundario al camarón y algunos peces cuando estos últimos se encuentran vedados. Específicamente, en la década pasada se reportó una disminución de casi $90 \%$ en las capturas de $H$. nigritus para la zona de pesca del noreste del Golfo de California (Cudney-Bueno, 2007). Las actuales estadísticas de extracción disponibles (2010-2017) para todas las especies de caracoles en el noroeste de México indican un incremento gradual (CONAPESCA, 2020; SEMARNAT, 2020), pero no proporcionan la captura por especie dejando un vacío de información en el potencial de recuperación ecológica de este caracol en la región. Por otro lado, esta especie no está categorizada en los registros de organismos protegidos (CITES, 2020), por lo que es considerada de interés en la planeación estratégica de pesquerías locales a baja escala de la parte norte del Golfo de California (Munguía-Vega, Torre, Turk-Boyer, Marinone, \& Lavin, 2015). Sin embargo, hasta ahora los reportes de su disminución se basan principalmente en la experiencia y opinión de los pescadores.

La información disponible acerca de su biología reproductiva que genere técnicas para su conservación y cultivo es escasa. Cudney-Bueno et al. (2008) describió la ecología reproductiva de una población silvestre del noreste del Golfo de California, mientras que Góngora-Gómez et al. (2011) y Camacho-Sánchez (2012) reportaron aspectos cuantitativos reproductivos de progenitores mantenidos en laboratorio, indicando que tanto la temperatura como la calidad del agua influyen en el desarrollo embrionario y larval. Los resultados del presente trabajo contribuyen con el conocimiento del desove y desarrollo intracapsular de $H$. nigritus en laboratorio, para aportar técnicas de manejo que ofrezcan estrategias en la conservación de este recurso.

\section{MATERIALES Y MÉTODOS}

Recolección de organismos: Los caracoles $(\mathrm{N}=18,9$ hembras y 9 machos) fueron recolectados a mano del sustrato arenoso-fangoso durante la marea baja en Bahía Navachiste $\left(25^{\circ} 22^{\prime}-25^{\circ} 35^{\prime} \mathrm{N} \& 108^{\circ} 42^{\prime}-108^{\circ} 56^{\prime} \mathrm{W}\right)$, Sinaloa, México, en abril 2017. Se limpiaron de epibiontes (algas, balanos y lapas) y lodo para después, ser transportados en recipientes de plástico (20 1) con agua de mar, al laboratorio de Malacología del IPN-CIIDIR-Sinaloa.

Sistema de recirculación de agua: Para mantener a los reproductores se construyó un sistema de recirculación de agua de flujo ascendente $(\approx 250 \mathrm{ml} / \mathrm{min})$, que consistió en: un contenedor de plástico de 2001 de capacidad (lleno con 1501 de agua de mar) que se usó como reservorio; un tanque de fibra de vidrio de 1501 de capacidad (lleno con 1001 de agua de mar) conteniendo capas de grava, arena, tul y fibra de vidrio separadas por tela mosquitero, las cuales, se inocularon con bacterias nitrificantes para que funcionara como filtro biológico; una red de tubos de PVC de $1 / 4$ de pulgada con orificios para distribuir el agua filtrada en el tanque de fibra de vidrio; y una bomba sumergible (Little Giant Pump Company) de $1 / 4$ HP con mangueras conectadas a la red de tubos de PVC. Para mantener la concentración de oxígeno disuelto mayor a $4 \mathrm{mg} / \mathrm{l}$, 


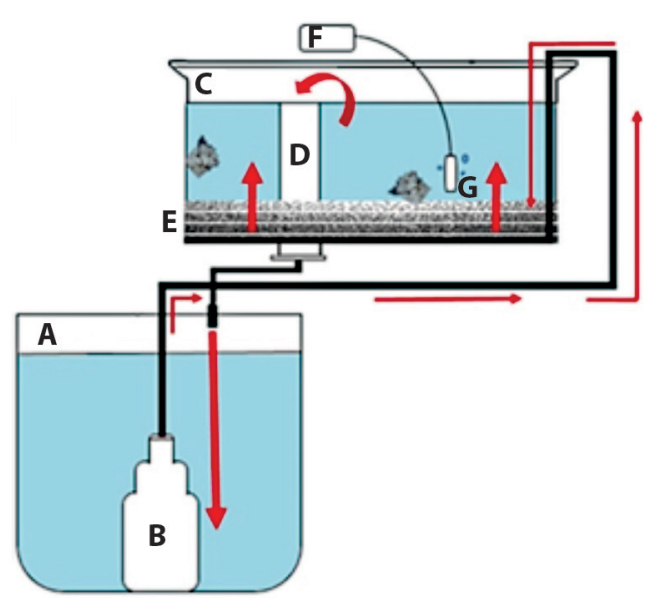

Fig. 1. Sistema de recirculación de agua de flujo ascendente para el mantenimiento de los reproductores de Hexaplex nigritus. A. Reservorio de agua (200 1); B. bomba sumergible; C. tanque de los caracoles (150 1); D. tubo de desagüe; E. capas de grava, arena, tul y fibra de vidrio separadas por tela mosquitero; F. aireador eléctrico; G. piedra de aireación. Las flechas indican el flujo de agua. Fig. 1. Upflow water recirculation system for the maintenance of Hexaplex nigritus broodstock. A. water reservoir (200 1); B. submersible pump; C. snail tank (150 1); D. drain pipe; E. gravel, sand, tulle, and fiberglass layers separated by mosquito netting; F. electric aerator; G. aeration stone. Arrows indicate the flow of water.

se introdujeron dos piedras de aireación al tanque de fibra de vidrio, conectadas a un soplador eléctrico de 5 HP. Se colocó una malla plástica encima del tanque para evitar la salida de los caracoles (Fig. 1).

Diariamente, se retiraban los desperdicios (heces y alimento) mediante sifoneo y se reponía el nivel con agua marina filtrada. Las paredes y tubos de distribución de agua se lavaban cada mes y se realizaba un recambio parcial de agua $(50 \%)$. Se cambiaba toda el agua del sistema cada dos meses o cuando algunos bivalvos que se proporcionaban como alimento morían y permanecían cerrados varios días, enturbiando el agua.

Aclimatación y mantenimiento de reproductores: Los caracoles se midieron y pesaron al inicio del estudio (longitud $=75.05$ $\pm 3.08 \mathrm{~mm}$, ancho $=49.59 \pm 2.01 \mathrm{~mm}$, peso $=$ $63.11 \pm 8.31 \mathrm{~g})$ y cada mes durante 18 meses (abril 2017-septiembre 2018), usando una regla Vernier $0.01 \mathrm{~mm}$, Mitutoyo 500-172) y una balanza granataria $(0.001 \mathrm{~g}$, Ohaus Scout Pro 2001), respectivamente. Los especímenes fueron colocados en el tanque de fibra de vidrio y alimentados ad libitum con varios bivalvos (Larkinia grandis, Chione fluctifraga, Megapitaria squalida, Mytella strigata, Dosinia ponderosa y Anadara mazatlanica) dependiendo en primera instancia, de la disponibilidad de estos y posteriormente, de la preferencia alimenticia mostrada por $H$. nigritus. Después de una semana, los caracoles mostraron un consumo regular de bivalvos, sugiriendo su adaptación a las condiciones de laboratorio (Güler \& Lök, 2014). Todos los días se registraron la temperatura, oxígeno disuelto (oxímetro YSI-55, Ohio 45387), salinidad (refractómetro, Atago S/Mill) y pH (potenciómetro Hanna HI 8314) del agua. Se mantuvo un fotoperiodo natural en todo el estudio.

Registro de desoves: Cada masa ovígera adherida a las paredes del tanque fue registrada y despegada cuidadosamente con un bisturí (Fig. 2); después, se enjuagaron con agua marina filtrada y el número de cápsulas por masa ovígera fue contabilizado. Se obtuvo una muestra de 18 cápsulas para ser medidas (longitud y ancho, mm, Cudney-Bueno et al., 2008; Mahmoud, Mohammed, \& Yassien, 2013) con una regla Vernier $(0.01 \mathrm{~mm})$ y pesadas con una balanza digital $(0.001 \mathrm{~g})$.

\section{Conteo de larvas (velígeras) por cápsu-} la: De una masa ovígera, se obtuvieron 8 cápsulas para contar el número de larvas velígeras que emergieron de cada una. Cada cápsula fue mantenida en un vial $(20 \mathrm{ml})$ hasta la ruptura de su ápice (Cudney-Bueno et al., 2008). Los viales fueron mantenidos a la temperatura ambiente del laboratorio durante el verano (28$30{ }^{\circ} \mathrm{C}$ ), cuando se presentaron los desoves, y se cambió el agua de los viales diariamente. De esta forma, se estimó el número total de larvas velígeras por cápsula y por masas ovígeras. 


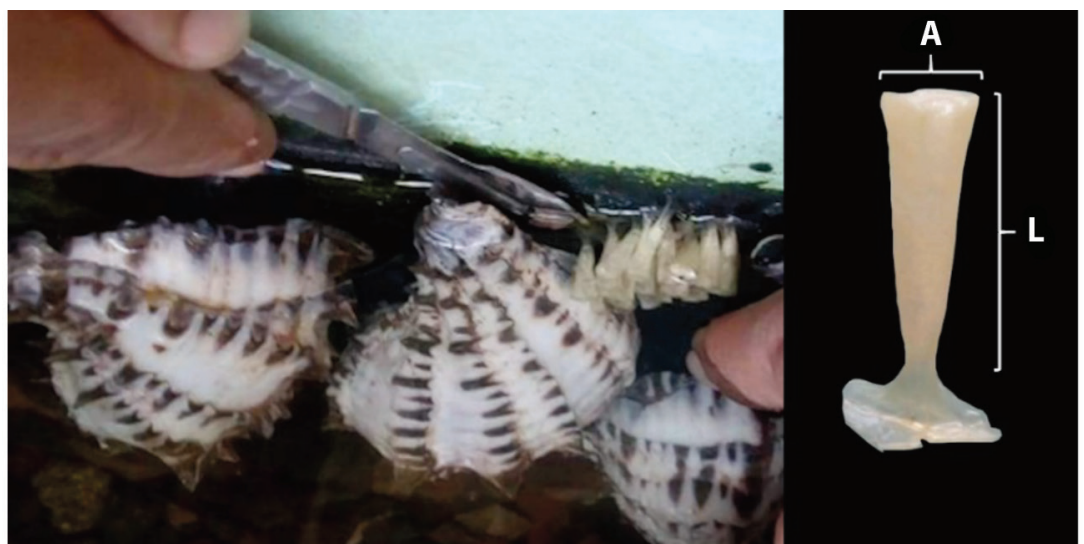

Fig. 2. Desprendimiento de la masa ovígera de Hexaplex nigritus del tanque experimental (izquierda) y dimensiones promedio $(\mathrm{L}=$ longitud, $\mathrm{A}=$ ancho, $\mathrm{mm}$ ) de la cápsula (derecha).

Fig. 2. Detachment of the ovigerous mass of Hexaplex nigritus from the experimental tank (left) and mean capsule dimensions ( $\mathrm{L}=$ length, $\mathrm{A}=$ width, $\mathrm{mm}$ ) (right).

Desarrollo embrionario y larval: Para describir el desarrollo embrionario (dentro de las cápsulas) y larvas (después del rompimiento del tapón o ápice de la cápsula y su expulsión) de $H$. nigritus, se colocaron 60 cápsulas de una masa ovígera en 3 peceras de vidrio ( $31, \mathrm{~N}=20$ cápsulas por pecera) y se mantuvieron en una incubadora con temperatura controlada (28.2 $\pm 1.5^{\circ} \mathrm{C}$ ) y aireación constante, mediante un calentador eléctrico (100 W, Visitherm) y una piedra de aireación en cada pecera, respectivamente. El oxígeno disuelto $(4.2 \pm 0.5 \mathrm{mg} / \mathrm{l}), \mathrm{pH}$ (7.81 \pm 0.12 unidades de $\mathrm{pH}, \mathrm{UpH}$ ) y la salinidad (33.41 \pm 2.47 unidades prácticas de salinidad, UPS) del agua fueron monitoreados cada día. Cada $8 \mathrm{~h}$ después de haber colocado las cápsulas en las peceras y durante los días hasta que su ápice se rompía, se extraía una cápsula de cada pecera, se cortaba cuidadosamente con un bisturí y en una caja de Petri, se colocaban los huevos viables o embriones para ser observados en un microscopio (10X y 40X, Wesco), fotografiarlos (cámara Nikon D5200 adaptada al microscopio) y describir sus estructuras. Al eclosionar las cápsulas y emergían las larvas plantónicas, se tomaba una muestra de $20 \mathrm{ml}$ para fotografiar y diferenciar las estructuras de los estadios larvales al microscopio. Se obtuvo la medida promedio $(\mu \mathrm{m})$ de embriones y larvas cada día con la ayuda de un micrómetro ocular. Desde el rompimiento del ápice hasta el asentamiento de las larvas, se alimentaron con una mezcla de microalgas (Isochrysis galbana, Chaetoceros gracilis y Tetraselmis suecica), como se indica en la Tabla 1.

La aparición de las estructuras embrionarias y larvales fue registrada y descrita con relación a su aparición en el tiempo, de acuerdo a Góngora (1999), Naegel \& Gómez del Prado-Rosas (2004) y Camacho-Sánchez (2009, 2012).

Estadísticos: Se analizó la normalidad y homogeneidad de la varianza de la cantidad de masas ovígeras mediante pruebas de ShapiroWilk y Brown-Forsythe, respectivamente. Se aplicó una prueba de Wilcoxon Mann-Whitney para evaluar si los datos provienen de una misma distribución o existen diferencias entre los muestreos. El análisis estadístico se realizó con un nivel de significancia de $95 \%$, usando RStudio, ver. 3.6.3 (R Core Team, 2018).

\section{RESULTADOS}

Los valores promedio de temperatura $\left(25.62 \pm 2.33{ }^{\circ} \mathrm{C}\right)$, salinidad $(34.62 \pm 2.68$ UPS), oxígeno disuelto $(5.09 \pm 0.57 \mathrm{mg} / \mathrm{l})$ 
TABLA 1

Régimen alimenticio de las larvas de Hexaplex nigritus

TABLE 1

Feeding regime of the Hexaplex nigritus larvae

$\begin{array}{cccc}\text { Día } & \begin{array}{c}\text { Suministro } \\ \text { de microalgas } \\ \text { (cél/ml) }\end{array} & \begin{array}{c}\text { Microalgas } \\ \text { consumidas } \\ \text { (cél/ml) }\end{array} & \text { Especies } \\ 1-14 & & & \\ 15-16 & & 5000 & \\ 17 & & 5000 & \\ 18 & & 12500 & \\ 19 & 45000 & 15000 & \text { Isochrysis galbana } \\ 20 & & 17500 & \text { y } \\ 21 & & 35000 & \text { Chaetoceros gracilis } \\ 22 & & 50000 & \\ 23 & & 67500 & \\ 24 & 90000 & 70000 & \\ 25 & & 75000 & \text { ND } \\ 26 & & 32500 & \text { I. galbana, } \\ 27 & 200000 & 10000 & \text { Ch. gracilis } \\ 28 & 150000 & \text { ND } & \text { Tetraselmis suecica } \\ 29 & & \text { ND } & \\ 30 & 100000 & & \\ 31 & & & \\ & & & \end{array}$

$\mathrm{ND}=$ No determinado.

y $\mathrm{pH}(7.61 \pm 0.36 \mathrm{UpH})$ del agua, se mantuvieron estables en el estudio. Los reproductores registraron un incremento en sus medidas durante los 18 meses en laboratorio (Fig. 3), con una tasa de crecimiento de $0.024 \mathrm{~mm} / \mathrm{d}$,
$0.027 \mathrm{~mm} / \mathrm{d}$ y $0.049 \mathrm{~g} / \mathrm{d}$, para la longitud, ancho y peso, respectivamente. Los valores finales para la longitud, ancho y peso fueron $82.99 \mathrm{~mm}, 57.97 \mathrm{~mm}$ y $76.82 \mathrm{~g}$, respectivamente. No se registró mortalidad.

Se observaron posturas de masas ovígeras en agosto y septiembre de ambos años. En 2017, se registraron 42 masas ovígeras adheridas a las paredes del tanque (17 en agosto y 25 en septiembre). La cantidad de cápsulas en agosto fluctuó de 25 a 228, mientras que en septiembre varió de 13 a 292. En 2018, se encontraron 39 masas ovígeras en los mismos meses (17 en agosto y 22 en septiembre), pero la cantidad de cápsulas presentó mayor fluctuación (23-455 en agosto y 11-514 en septiembre).

Los valores promedio de la longitud, ancho y peso de cada cápsula $(\mathrm{N}=18)$ fueron $14.07 \pm 0.76 \mathrm{~mm}, 4.40 \pm 0.32 \mathrm{~mm}$ y $0.044 \pm$ $0.006 \mathrm{~g}$, respectivamente. Las cápsulas presentaron una apariencia cónica con el ápice en el extremo superior (Fig. 4). En algunas masas ovígeras se detectaron cápsulas de color blanquecino, morado y pocas blancas, pero mal formadas (Fig. 4).

Del total de cápsulas recolectadas $(\mathrm{N}=11$ 465), $94.39 \%$ fueron viables, $5.27 \%$ resultaron de color morado y $0.34 \%$ presentaron malformaciones (Tabla 2). En estas dos últimas no se desarrollaron embriones (no viables). No

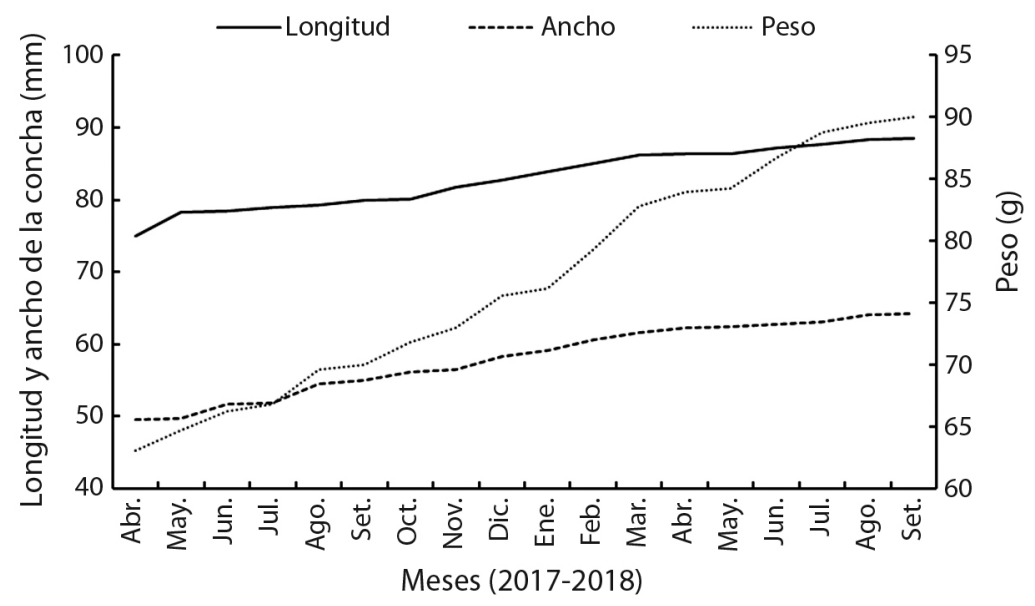

Fig. 3. Longitud, ancho ( $\mathrm{mm}$ ) y peso (g) de Hexaplex nigritus en condiciones de laboratorio.

Fig. 3. Length, width (mm), and weight $(\mathrm{g})$ of Hexaplex nigritus in laboratory conditions. 

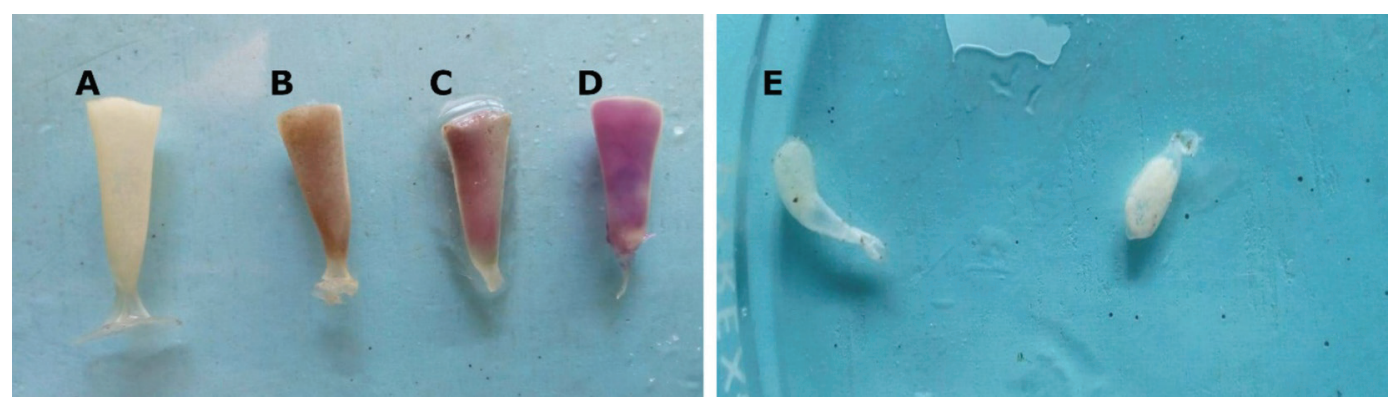

Fig. 4. Apariencia de cápsulas viables (A. fertilizadas, B. y C. en desarrollo embrionario) y no viables (D. moradas y E. malformadas).

Fig. 4. Appearance of viable (A. fertilized, B. and C. in embryonic development and non-viable D. purple, E. malformed capsules).

TABLA 2

Cápsulas viables y no viables (moradas y malformadas) en las masas ovígeras de Hexaplex nigritus

TABLE 2

Viable and non-viable (purple and malformed) capsules in the ovigerous masses of Hexaplex nigritus

\begin{tabular}{lccc} 
& 2017 & 2018 & Total \\
Cápsulas totales & 5057 & 6408 & 11465 \\
Cápsulas viables & 4745 & 6077 & 10822 \\
Cápsulas moradas & 294 & 310 & 604 \\
Cápsulas malformadas & 18 & 21 & 39 \\
\hline
\end{tabular}

se encontraron diferencias significativas ( $\mathrm{W}=$ 95.0, $\mathrm{P}=0.37)$ en la producción de cápsulas por año.

El número promedio de larvas velígeras por cápsula $(\mathrm{N}=8)$ fue de $1059.50 \pm 83.09$.

En la Fig. 5 se describe el desarrollo intracapsular de los embriones del caracol $H$. nigritus, destacando la aparición gradual desde el cuerpo polar en el huevo fertilizado, hasta los cilios, riñones laterales, protuberancia cefálica, lóbulos velares, ojos, corazón, opérculo y concha con espinas, de la larva pre-velígera.

En la Fig. 6 se describe el desarrollo larval de $H$. nigritus después del rompimiento del ápice capsular, destacando la presencia gradual de la torsión de la concha, estatocitos, gónada, pie, canal sifonal y primera y segunda volutas de la concha.

La Tabla 3 indica la longitud promedio $(\mu \mathrm{m})$ y tiempo de desarrollo de las estructuras morfológicas de las fases de embrión y larva lecitotrófica intracapsular y larva planctónica de $H$. nigritus, desde el desove hasta el asentamiento larvario. El desarrollo desde el huevo fertilizado $(110 \mu \mathrm{m})$ hasta la eclosión de la larva velígera $(301 \mu \mathrm{m})$ tuvo una duración de 14-15 días, mientras que la larva asentada (730 $\mu \mathrm{m})$ se registró al día 31-32. La concha y espinas fueron diferenciadas al día 8 de desarrollo. Las volutas de la concha larval se distinguieron el día 21 de desarrollo cuando la larva velígera alcanzó una longitud de $570 \mu \mathrm{m}$.

La Tabla 4 muestra características comparativas del desarrollo intracapsular y larval de Hexaplex nigritus y diferentes especies de murícidos. El diámetro del huevo entre diferentes especies fluctuó de 110 a 280-355 $\mu \mathrm{m}$, el primer valor obtenido en el presente estudio. Todas las características del desarrollo intracapsular y larval de las especies (diámetros del huevo, longitud y días en alcanzar el estado de larva velígera, tiempo de asentamiento larval y temperatura de incubación) mostraron valores diferentes entre sí.

\section{DISCUSIÓN}

Dentro del grupo de los caracoles murícidos, la formación de masas ovígeras compuestas de cápsulas en las que se desarrollan embriones y larvas representa una de las características reproductivas más sobresalientes (Romero, Gallardo, \& Bellolio, 2004; 

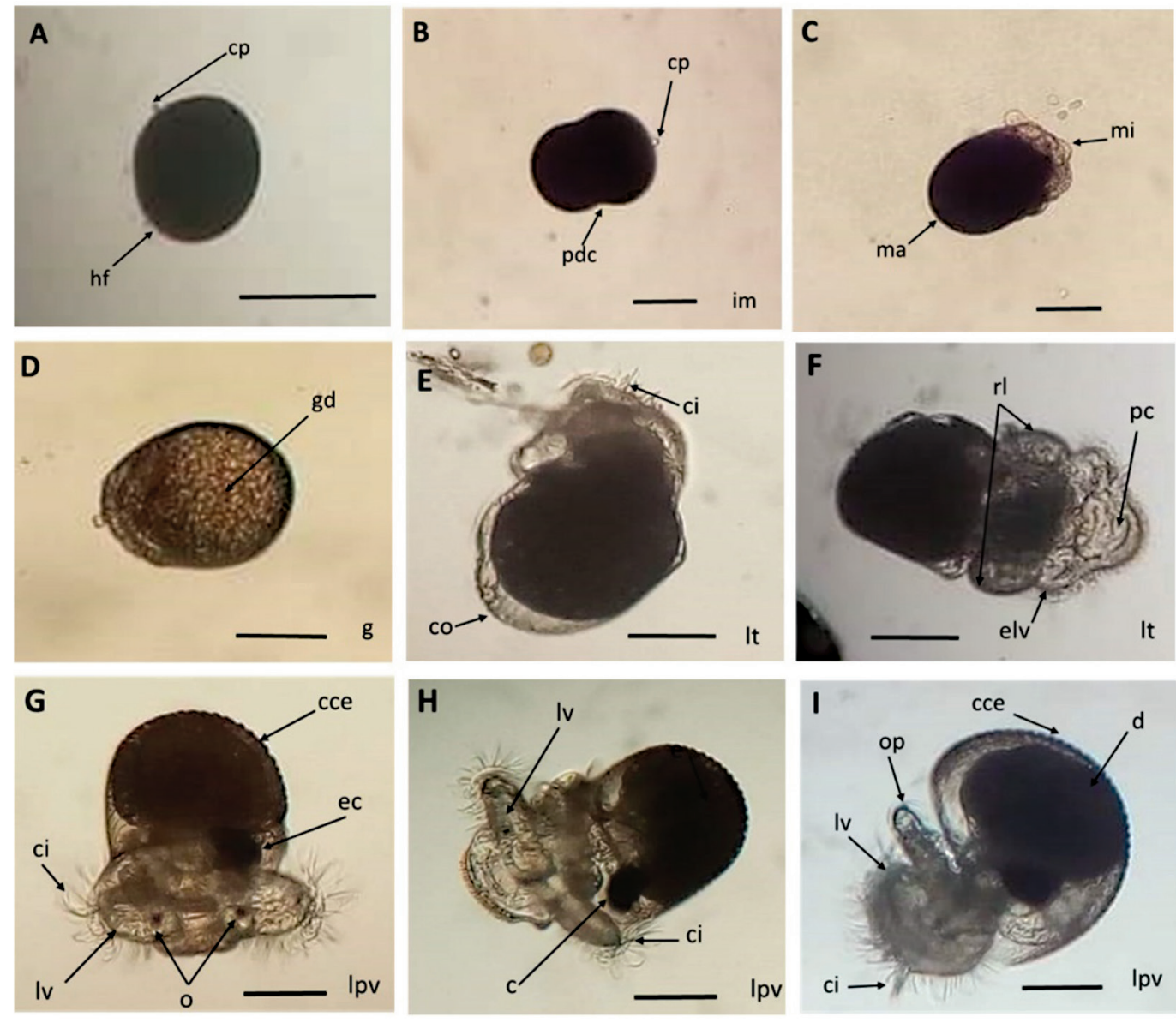

Fig. 5. Desarrollo embrionario intracapsular de Hexaplex nigritus. A. Huevo fertilizado antes de la primera división celular. B. Primera división celular. C. Mórula. D. Gástrula. E. Larva trocófora temprana. F. Aparición de riñones larvarios. G. Larva pre-velígera temprana. H. Larva pre-larva velígera avanzada. I. Larva pre-velígera a punto de eclosionar. Abreviaciones: $\mathrm{c}=$ corazón, $\mathrm{cce}=$ concha con espinas, $\mathrm{ci}=$ cilios, $\mathrm{co}=$ concha, $\mathrm{cp}=$ cuerpo polar, $\mathrm{d}=$ digestivo, ec $=$ esbozo de corazón, elv = esbozo de lóbulos velares, $\mathrm{g}=$ gástrula, $\mathrm{gd}=$ glándula digestiva, $\mathrm{hf}=$ huevo fertilizado, $\mathrm{im}=$ inicio mórula, $\mathrm{lt}=$ larva trocófora, $\mathrm{lv}=$ lóbulos velares, $\mathrm{lpv}=$ larva pre-velígera, $\mathrm{ma}=$ macrómero, $\mathrm{mi}=$ micrómero, $\mathrm{o}=\mathrm{ojos}, \mathrm{op}=\mathrm{opérculo}, \mathrm{pc}=$ protuberancia cefálica, $\mathrm{pdc}=$ primera división celular, $\mathrm{rl}=$ riñones larvarios. Escala: $100 \mu \mathrm{m}$.

Fig. 5. Intracapsular embryonic development of Hexaplex nigritus. A. Fertilized egg before the first cell division. B. First cell division. C. Morula. D. Gastrula. E. Early trocophore larva. F. Appearance of larval kidneys. G. Early pre-veliger larva. H. Advanced pre-veliger larva. I. Nearly-hatched pre-veliger larva. Abbreviations: $\mathrm{c}=$ heart, $\mathrm{cce}=$ shell with spines, $\mathrm{ci}=$ cilia, $\mathrm{co}=$ shell, $\mathrm{cp}=$ polar body, $\mathrm{d}=$ digestive, $\mathrm{ec}=$ early heart, elv = early velar lobes, $\mathrm{g}=$ gastrula, $\mathrm{gd}=$ digestive gland, $\mathrm{hf}=$ fertilized egg, $\mathrm{im}=$ early morula, $\mathrm{lt}=$ trochophore larva, $\mathrm{lv}=$ velar lobes, $\mathrm{lpv}=$ pre-veliger larva, $\mathrm{ma}=$ macromer, $\mathrm{mi}=$ micromere, $\mathrm{o}=$ eyes, op $=$ cephalic protuberance, $\mathrm{pdc}=$ first cell division, $\mathrm{rl}=$ larval kidneys. Scale: $100 \mu \mathrm{m}$.

Pastorino, Penchaszadeh, \& Scarabino, 2007; Cudney-Bueno et al., 2008), lo que facilita el estudio de aspectos biológicos como su potencial reproductivo (Pechenik, Chang, \& Lord, 1984), desarrollo embrionario (Cumplido, Pappalardo, Fernández, Averjub, \& Bigatti,
2011) y nutrición intracapsular (Güler \& Lök, 2014), entre otros, ya sea mediante la recolecta de masas ovígeras del medio natural o la obtención de las mismas manteniendo reproductores en laboratorio. Aunque la recolecta de masas ovígeras directamente del medio natural 

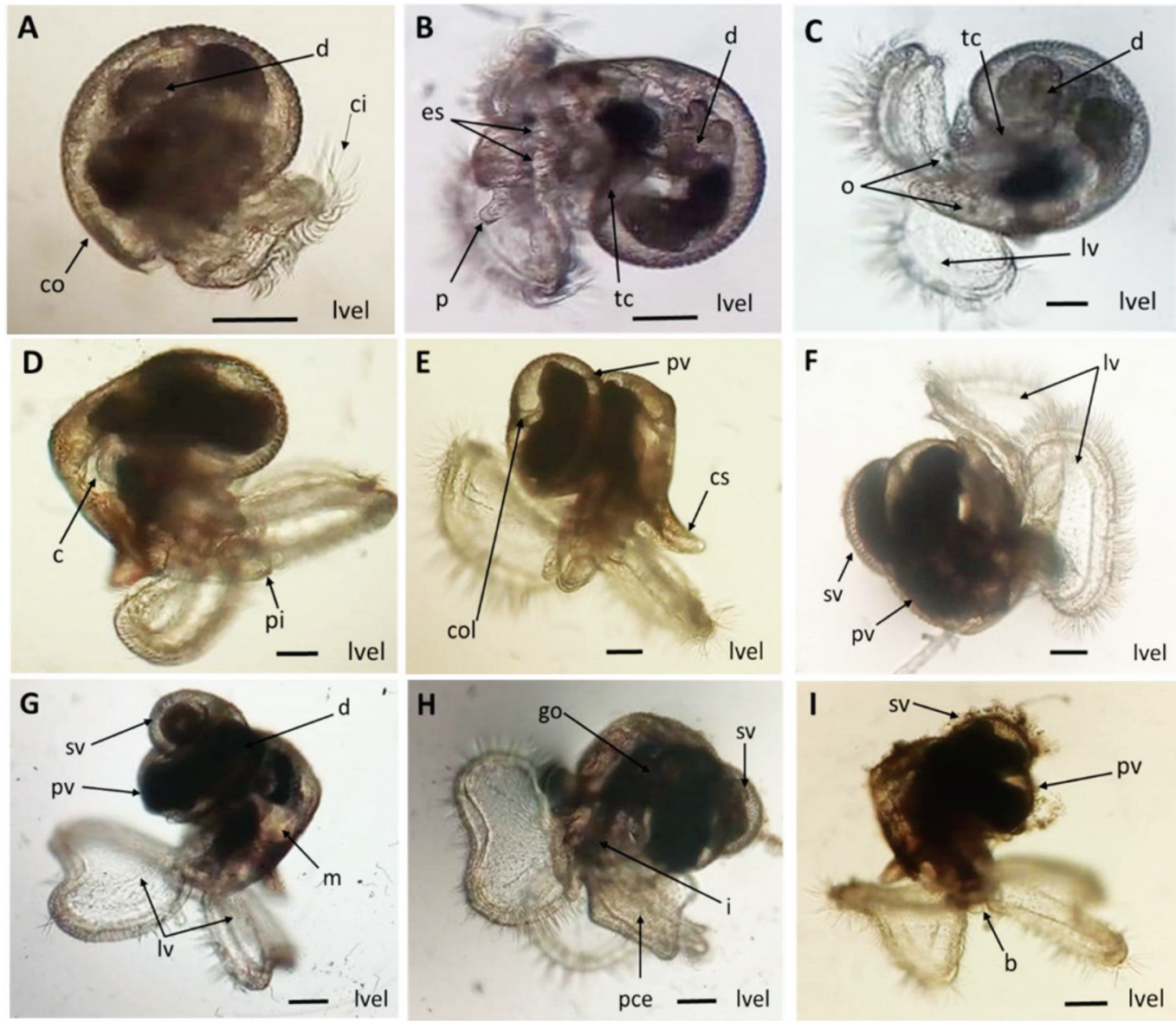

Fig. 6. Desarrollo larval de Hexaplex nigritus. A-I. Larva velígera. Abreviaciones: $\mathrm{b}=\mathrm{b}$ bca, $\mathrm{c}=\mathrm{corazón}$, $\mathrm{ci}=\mathrm{cilios}$, $\mathrm{co}=$ concha, col = columela, $\mathrm{cs}=$ canal sifonal, $\mathrm{d}=$ digestivo, es $=$ estatocitos, go = gónada, $\mathrm{i}=$ intestino, $\mathrm{lv}=$ lóbulos velares, lvel $=$ larva velígera, $\mathrm{m}=$ manto, $\mathrm{o}=\mathrm{ojos}, \mathrm{p}=$ probóside, $\mathrm{pce}=$ pie ciliado con espinas, $\mathrm{pi}=\mathrm{pie}, \mathrm{pv}=$ primera voluta, $\mathrm{sv}=$ segunda voluta, tc $=$ torsión de la concha. Escala: $100 \mu \mathrm{m}$.

Fig. 6. Larval development of Hexaplex nigritus. A-I. Veliger larva. Abbreviations: $\mathrm{b}=$ mouth, $\mathrm{c}=\mathrm{heart}, \mathrm{ci}=\mathrm{cilia}$, co $=$ conch, $\mathrm{col}=$ columella, $\mathrm{cs}=$ siphonal canal, $\mathrm{d}=$ digestive, es = statocytes, go = gonad, $\mathrm{i}=$ intestine, $1 \mathrm{v}=$ velar lobes, lvel = veliger larva, $\mathrm{m}=$ mantle, $\mathrm{o}=$ eyes, $\mathrm{p}=$ proboscis, $\mathrm{pce}=$ ciliated foot with spines, $\mathrm{pi}=$ foot, $\mathrm{pv}=$ first volute, $\mathrm{sv}=\mathrm{second}$ volute, tc $=$ torsion of the shell. Scale: $100 \mu \mathrm{m}$.

disminuye el tiempo experimental en laboratorio (González \& Gallardo, 1999), no permite conocer la etología de los reproductores en cautiverio (Zupo \& Patti, 2009) ni su capacidad reproductiva (Jagadis, Shunmugasundaram, Sathakkathulla, \& Mohanraj, 2013). En el presente trabajo, el caracol H. nigritus exhibió una rápida adaptación fisiológica a las condiciones del sistema experimental, coincidiendo con las observaciones de trabajos previos con la misma especie (Góngora-Gómez et al., 2011;
Camacho-Sánchez, 2012) e, inclusive, aumentando el tiempo de confinamiento a dos años, sin presentar mortalidad.

Estudios previos reportan el uso de diferentes tipos de alimento (vivo e inerte) en el mantenimiento de progenitores de gastrópodos marinos (Naegel \& Gómez del Prado-Rosas, 2004; Góngora-Gómez, Domínguez-Orozco, Muñoz-Sevilla, \& Rodríguez-Gil, 2007; Smith \& Thatje, 2013), lo cual, sugiere una rápida y fácil adaptación al régimen alimenticio 
TABLA 3

Longitud $(\mu \mathrm{m})$ y tiempo de desarrollo de las estructuras morfológicas de las etapas de embrión intracapsular lecitotrófico y larva planctónica de Hexaplex nigritus en laboratorio

TABLE 3

Length $(\mu \mathrm{m})$ and development time of the morphological structures of the stages of lecithotrophic intracapsular embryo and planktonic larva of Hexaplex nigritus in the laboratory

\begin{tabular}{lcc}
\multicolumn{1}{c}{ Estructuras morfológicas } & Longitud $(\mu \mathrm{m})$ & Tiempo de desarrollo (d) \\
Huevo fertilizado & 110 & $24 \mathrm{hr}$ \\
Macrómeros y micrómeros & 195 & $48-72 \mathrm{hr}$ \\
Inicio del movimiento & 195 & Día 4 \\
Gastrulación & 220 & Día 5 \\
Movimientos más notorios & 255 & Día 6 \\
Cilios & 255 & Día 7 \\
Concha y espinas & 255 & Día 8 \\
Lóbulos velares y protuberancia cefálica & 270 & Día 9 \\
Esbozo de corazón y ojos & 275 & Día 10 \\
Opérculo & 295 & Día 11 \\
Larva pre-velígera & 295 & Día 12-13 \\
Eclosión, larva-velígera & 310 & Día 14-15 \\
Probóscide, estatocistos y torsión de la concha & 340 & Día 16-19 \\
Pie & 550 & Día 20 \\
Primera voluta, columela y canal sifonal & 570 & Día 21 \\
Segunda voluta & 630 & Día 22-23 \\
Intestino, gónada y manto & $670-710$ & Día 24-26 \\
Desarrollo de todas las estructuras & $710-730$ & Día 27-29 \\
Boca larval & 730 & Día 30 \\
Asentamiento larval & 730 & Día 31-32 \\
\hline
\end{tabular}

TABLA 4

Parámetros de desarrollo embrionario intracapsular y larval de especies de murícidos

TABLE 4

Parameters of intracapsular and larval embryonic development of muricidae species

\begin{tabular}{|c|c|c|c|c|c|c|}
\hline Especie & $\begin{array}{l}\text { Diámetro del } \\
\text { huevo }(\mu \mathrm{m})\end{array}$ & $\begin{array}{c}\text { Larva } \\
\text { velígera }(\mu \mathrm{m})\end{array}$ & $\begin{array}{c}\text { Larva } \\
\text { velígera }(\mathrm{d})\end{array}$ & $\begin{array}{l}\text { Asentamiento } \\
\text { (d) }\end{array}$ & $\begin{array}{l}\text { Temperatura } \\
\left({ }^{\circ} \mathrm{C}\right)\end{array}$ & Autor \\
\hline Plicopurpura pansa & 149 & 188 & $19-47$ & $35-65$ & $21-23$ & $\begin{array}{l}\text { Naegel y Gómez del Prado- } \\
\text { Rosas, } 2004\end{array}$ \\
\hline Hexaplex trunculus & 235 & 375 & $20-29$ & $34-43$ & $18-22$ & Güler y Lök, 2014 \\
\hline Chicoreus ramosus & $280-355$ & $1150-1220$ & 29 & 40 & $19-24$ & Mahmoud et al., 2013 \\
\hline Chorus giganteus & 249 & 1100 & $59-72$ & $\begin{array}{l}\text { 24-72 hr después } \\
\text { de eclosión }\end{array}$ & 15.5 & González y Gallardo, 1999 \\
\hline Trophon geversiarus & 270 & 1400 & $20-30$ & $100-120$ & $12-14$ & Cumplido et al., 2011 \\
\hline Buccinum undatum & 230 & 1650 & $42-77$ & $133-140$ & 6 & Smith y Thatje, 2013 \\
\hline Hexaplex nigritus & 192 & 334 & $10-11$ & $17-19$ & $30-34$ & Camacho-Sánchez, 2012 \\
\hline Hexaplex nigritus & 110 & 310 & $14-15$ & $31-32$ & 28 & Este estudio \\
\hline
\end{tabular}


en condiciones controladas. Lo anterior coincide con nuestras observaciones, ya que los reproductores de $H$. nigritus consumieron diferentes especies de bivalvos vivos como lo observado por Góngora-Gómez et al. (2011). Sin embargo, a pesar de que en ocasiones se proveyó de almejas del género Chione, almejas voladoras (Argopecten circularis, A. ventricosus, Argopecten sp.), mejillón (Mytella strigata), almeja chocolata (Megapitaria squalida) y almejas pata de mula (Larkinia grandis y Anadara mazatlanica), los murícidos presentaron mayor preferencia por estas dos últimas cuando se suministró una mezcla de varias especies.

Con relación al desove, los caracoles colocaron masas ovígeras en los mismos meses de cada año, sugiriendo que su periodo de reproducción fue consistente con las condiciones mantenidas en el laboratorio, pero dictado ya sea por la estacionalidad del fotoperiodo natural (Wayne, 2001), un reloj endócrino (Fabioux, Huvet, Le Souchu, Le Pennec, \& Pouvreau, 2005) o ambos. De hecho, mostraron el mismo patrón para escoger el lugar de desove dentro del tanque en los dos años de estudio. Es decir, las condiciones ajustadas en el sistema cerrado de cultivo fueron adecuadas para propiciar la respuesta reproductiva de los gastrópodos. Por lo anterior, la fácil adaptación y el comportamiento reproductivo de $H$. nigritus en el laboratorio sugieren que la producción masiva de esta especie es una opción viable para su repoblación y conservación.

Existen reportes que indican una relación positiva entre la talla de los reproductores y el número de crías producidas por desove en invertebrados marinos. Específicamente en gastrópodos, Chatzinikolaou y Richardson (2010) documentaron que, especímenes del buccino Nassarius reticulatus con una talla promedio de $28 \mathrm{~mm}$ produjeron en laboratorio, hasta seis veces más cápsulas que caracoles con una longitud de la concha promedio de $22 \mathrm{~mm}$; mientras que Cañete, Gallardo, Céspedes, Cárdenas, \& Santana (2012) obtuvieron una proporción lineal entre el tamaño de la hembra y el número de cápsulas producidas por el caracol ranélido Fusitriton magellanicus. Sin embargo,
Schwab y Allen (2014) no encontraron diferencias en la cantidad de cápsulas producidas por dos clases de talla (15-19 y 21-25 mm) del caracol del lodo, Ilyanasa obsoleta $(=$ Tritia obsoleta), pero los especímenes de mayor longitud produjeron más huevos al interior de las cápsulas. Lo anterior puede ser explicado por la mayor cantidad de reservas energéticas que acumulan los organismos más grandes (Chaparro y Flores, 2002), y consecuentemente, en mayor cantidad de crías producidas.

En el presente estudio, la respuesta reproductiva del grupo de progenitores de $\mathrm{H}$. nigritus fue similar en ambos años, a pesar de la diferencia en la longitud de su concha y peso corporal que adquirieron en confinamiento y después de dos eventos reproductivos, uno por año. En promedio, los especímenes que desovaron en agosto y septiembre de 2017 (longitud $=79.6 \pm 3.13 \mathrm{~mm}$, ancho $=54.75 \pm$ $3.75 \mathrm{~mm}$, peso $=69.75 \pm 9.81 \mathrm{~g}$ ) colocaron 42 masas ovígeras, mientras que un año después, se encontraron adheridas 39 masas ovígeras cuando los caracoles eran más grandes (longitud $=88.37 \pm 4.46 \mathrm{~mm}$, ancho $=64.10 \pm 4.35$ $\mathrm{mm}$, peso $=89.94 \pm 15.20 \mathrm{~g}$ ). En este caso, la producción de cápsulas anual no presentó diferencias $(\mathrm{W}=95.0, \mathrm{P}=0.37)$ a pesar de que, en 2018, los caracoles fueron casi $10 \mathrm{~mm}$ más grandes y $20 \mathrm{~g}$ más pesados que al inicio del estudio. Dame (1996) indica que individuos jóvenes reproductivos de una población tienden a asimilar energía para el crecimiento somático, mientras que adultos priorizan la formación de tejido reproductivo. La similitud en la respuesta reproductiva de los progenitores aún con una diferencia de casi $10 \mathrm{~mm}$ en la longitud, sugiere que los caracoles, ya maduros sexualmente, eran aún jóvenes. Es decir, la talla de las hembras no varió lo suficiente como para observar cambios en su capacidad de reproducción, coincidiendo con Camacho-Sánchez (2012) para la misma especie. Las diferencias en los resultados con otros gastrópodos pudieran ser parcialmente explicadas por sus características intrínsecas (Ito, 1997), además de las condiciones experimentales de cada trabajo. 
La cápsula de huevos de los caracoles murícidos varía de acuerdo a su forma (D’Asaro, 1992). La descripción de las cápsulas de $H$. nigritus coincide con las detalladas para Murex florifer (D'Asaro, 1970), Priene scabrum (Romero, Lohrmann, Bellolio, \& Dupre, 2003) y Fasciolaria tulipa (Moffa, Chávez, Bastardo, Villalba, \& Crescini, 2017). La coloración de las cápsulas se oscureció hasta tornarse de color café conforme los embriones se desarrollaban, sin embargo, se observaron algunas cápsulas malformadas (en forma de gota, translúcidas y flexibles o blandas, sin firmeza) y otras de color morado, ambas con embriones muertos. La observación de embriones muertos en cápsulas moradas de $H$. nigritus coincide con el reporte de Naegel y Gómez del Prado-Rosas (2004) para Plicopurpura pansa. En contraste, la transición de coloración capsular de blanco a morado es parte normal de la biología reproductiva del murícido australiano Dicathais orbita, dada por la conversión gradual de compuestos antimicrobianos -(tirindoleninona y tiriverdina, que protegen a los embriones de infecciones durante su desarrollo)-, en púrpura de Tiro, el cual, carece de estas propiedades (Benkerdorff, Bremner, \& Davis, 2000).

Similarmente, $H$. nigritus presenta cambios graduales de coloración capsular, aunque normalmente sin llegar a un estadío morado. Aunque la presencia de estos compuestos antimicrobianos no ha sido estudiada en $H$. nigritus, la producción de púrpura de Tiro observada en múltiples miembros de la familia Muricidae (Naegel \& Cooksey, 2002; Benkerdorff, Westley, \& Gallardo, 2004), junto con los cambios graduales de coloración similares a $D$. orbita y la conversión de un $5.27 \%$ de cápsulas al estadío morado, sugieren su presencia en las cápsulas de $H$. nigritus. Considerando el riesgo de infección bacteriana presente en cautiverio (D’Asaro, 1966; Riquelme \& Chávez, 1991), la pérdida de actividad antimicrobiana en algunas cápsulas de $H$. nigritus puede explicar la mortalidad de sus embriones. Sin embargo, no se sabe si la producción de un porcentaje de cápsulas moradas es normal en $H$. nigritus o es consecuencia de algún aspecto del cautiverio.

Las anteriores observaciones proveen nuevos objetivos para posteriores estudios de la biología y cultivo este caracol. Acorde a nuestro conocimiento, no existen descripciones de cápsulas malformadas no viables parecidas a las encontradas en este estudio, por lo que no es posible discutir la causa por la que se originaron. Sin embargo, la flexibilidad y transparencia mostradas en las cápsulas malformadas sugieren una posible falta de sales y proteínas (Rawlings, 1995) incorporadas a las capas de la cápsula que aumentan su firmeza y permiten las funciones de transporte de solutos y gases, además de evitar el estrés térmico y por desecación (Lord, 1986; Buckland-Nicks \& Chia, 1990; Fu, Guerette, Tan, Zhao, \& Schefer, 2015), algo que pudiera estar relacionado con la variedad, calidad y cantidad del alimento suministrado, pero que debe ser estudiado más profundamente.

Días antes de que comenzaran a desovar cada año, $H$. nigritus mostró una reducción en su alimentación, lo que coincide con las observaciones de Brokordt (2003) y Güler y Lök (2014) para Buccinum undatum y Hexaplex trunculus, respectivamente, en laboratorio. Este fenómeno es común en moluscos marinos como un procedimiento preparativo de la reproducción, en el que la gónada crece de tamaño gracias a las reservas alimenticias transferidas desde otros tejidos (Bayne, 1976; Bayne \& Newell, 1983; Navarro, Leiva, Gallardo, \& Varela, 2002). El promedio de cápsulas por masa ovígera $(\mathrm{N}=141)$ fue similar al reportado para la misma especie en laboratorio ( $\mathrm{N}=137$, Cudney-Bueno et al., 2008; $\mathrm{N}=134$, Góngora-Gómez, García-Ulloa, \& Domínguez, 2010; N = 150, Góngora-Gómez et al., 2011; N = 133, Camacho-Sánchez, 2012), pero difirió de Chorus giganteus (González \& Gallardo, 1999), Chicoreus ramosus (Mahmoud et al., 2013) y Hexaplex trunculus (Güler y Lök, 2014) $(\mathrm{N}=4,101$ y 108, respectivamente). Lo anterior puede explicarse por factores intrínsecos a cada especie, como su genética y tamaño, al igual que las condiciones de laboratorio. 
No se obtuvo el número total de huevos fertilizados por cápsula, ni la comparación de estos con el número de larvas velígeras después del rompimiento del ápice capsular, por lo que no se puede discutir acerca de la posible presencia de huevos nutricios intracapsulares. Sin embargo, la cantidad promedio de larvas velígeras de $H$. nigritus registradas después del rompimiento de la cápsula, presumiría que, dentro de dicha estructura, se alimentan de las reservas vitelinas transferidas por la hembra al huevo (lecitotróficos), coincidiendo con las observaciones de Naegel y Gómez del Prado-Rosas (2004) para Plicopurpura pansa y Cudney-Bueno et al. (2008) con el murícido del presente trabajo.

La cantidad de larvas eclosionadas en el presente trabajo fue menor al reportado por Cudney-Bueno et al. (2008) para H. nigritus. Es de notar que estos autores utilizaron ejemplares más grandes en longitud (123.3 \pm $4.3 \mathrm{~mm}$ ), mismos que produjeron cápsulas de mayor tamaño $(16.23 \mathrm{~mm}) \mathrm{y}$, en consecuencia, más del triple de larvas (3 $603 \pm 221)$ que las que se obtuvieron en el presente trabajo (1 $059.5 \pm 83.09)$. La relación entre la talla de los reproductores, principalmente la hembra, y la capacidad reproductiva mencionada antes (Chatzinikolaou \& Richardson, 2010; Cañete et al., 2012), refuerza la diferencia en estos resultados. Por año, es posible asumir que cada hembra $(\mathrm{N}=9)$ de $H$. nigritus con un rango de longitud de 80-88 mm, es capaz de producir hasta 4 masas ovígeras, 627 cápsulas y 663993 larvas veliger extracapsulares. Este potencial reproductivo es significativo cuando se trata de su producción en laboratorio con fines de repoblación y conservación.

El diámetro promedio de los huevos fecundados fue menor no sólo con los registrados para otros murícidos, sino también, con el reportado por Camacho-Sánchez (2012) para la misma especie del presente estudio (Tabla 4); lo anterior sugiere un desarrollo embrionario más rápido al ser dependiente de la cantidad de reservas vitelinas, que se confirma también, con el tiempo reducido (14-15 d) de incubación intracapsular mostrado, y posteriormente, con los 31-32 d requeridos para alcanzar el estadio de juvenil metamorfizado y asentado. Este mismo patrón en las diferencias morfométricas entre las especies se mantuvo al comparar la talla de la larva velígera. La táctica reproductiva de $H$. nigritus, característica de una estrategia tipo " $r$ ", es decir, basada en la producción de muchos huevos de menor tamaño, coincide con las observaciones de Barnes (1990) al contrastar poblaciones del caracol Hydrobia ulvae, lo cual, garantizaría la supervivencia de juveniles metamorfizados en su hábitat. Sin embargo, serían pocos los adultos que alcanzarían la madurez reproductiva. En condiciones controladas, la supervivencia de juveniles es igualmente mayor, pero puede ser favorecida con una pre-engorda que sirva para criar más juveniles con mayores probabilidades de reproducción (Najmudeen \& Victor, 2004; Jagadis, Kavitha, Padmanathan, Maharshi, \& Varadarajakumar, 2017).

Es bien reconocido en gastrópodos marinos que el tiempo de desarrollo larvario es afectado por la temperatura (Pechenik \& Lima, 1984; Lima \& Pechenik, 1985). En el presente estudio, la temperatura en el desarrollo intracapsular larvario de $H$. nigritus $\left(28{ }^{\circ} \mathrm{C}\right)$ fue superior al reportado para otras especies (Tabla 4), coincidiendo con la reducción en el tiempo del desarrollo embrionario y larval. Más aún, el rango de temperatura $\left(30-34{ }^{\circ} \mathrm{C}\right)$ documentado por Camacho-Sánchez (2012) para esta misma especie, redujo casi la mitad de dicho tiempo comparado con nuestros resultados, sugiriendo que este caracol puede desarrollarse en un amplio rango de temperatura.

A pesar de que este murícido exhibió una fácil y rápida adaptación a las condiciones generales de cultivo y una respuesta reproductiva bien definida estacionalmente, pudieron detectarse algunos aspectos de operación experimental que, aunque no afectaron los resultados, es necesario considerar en futuros trabajos. Por ejemplo, se observó la presencia de protozoarios filamentosos adheridos en las larvas después del rompimiento del ápice capsular, lo cual, fue controlado con el recambio de agua. Así mismo, las almejas muertas 
que permanecían en el tanque al menos durante una noche, se descomponían y ensuciaban el agua de la unidad experimental, haciendo necesario el cambio de toda el agua del sistema. Por lo anterior, se recomienda la renovación de almejas provistas como alimento cada noche para evitar el posible deterioro de la calidad de agua. También, el marcaje de los especímenes para conocer el potencial reproductivo individual (cantidad exacta de cápsulas de acuerdo a la talla de caracol estudiada), representaría una útil herramienta. Finalmente, el presente estudio aporta conocimientos para establecer condiciones en laboratorio (sustrato, parámetros, etc.), crecimiento y manejo de reproductores, alimentación, aprovisionamiento de juveniles, potencial reproductivo y aparición de estructuras embrionarias y larvarias del caracol murex negro chino Hexaplex nigritus, que representan útiles herramientas para su posible conservación y protección mediante técnicas acuícolas, inclusive, para fines de repoblación.

Declaración de ética: los autores declaran que todos están de acuerdo con esta publicación y que han hecho aportes que justifican su autoría; que no hay conflicto de interés de ningún tipo; y que han cumplido con todos los requisitos y procedimientos éticos y legales pertinentes. Todas las fuentes de financiamiento se detallan plena y claramente en la sección de agradecimientos. El respectivo documento legal firmado se encuentra en los archivos de la revista.

\section{AGRADECIMIENTOS}

Los autores agradecen al Instituto Politécnico Nacional por el apoyo logístico y económico otorgado para el desarrollo de esta investigación mediante los proyectos IPNSIP 20200527 and IPNSIP 20200526. Misael Pinzón Zúñiga reconoce a la Universidad Autónoma de Occidente, Unidad Guasave, y al Laboratorio de Malacología del Centro Interdisciplinario de Investigación para el Desarrollo Integral Regional, CIIDIR-Sinaloa por facilitarle lo necesario para culminar con su tesis de Licenciatura en Biología. Un agradecimiento especial se extiende a Jesús Humberto Heredia Bacasegua por proveer las microalgas y asesorar, tanto en su mantenimiento, como en la supervisión del cultivo larvario del caracol.

\section{RESUMEN}

Introducción: El caracol marino Hexaplex nigritus es un murícido fuertemente explotado en el Golfo de California para consumo y artesanías. Cuando se reproducen, los adultos se agregan en forma de arrecifes artificiales facilitando su identificación y extracción, lo cual, ha mermado su población en dicha zona. Objetivo: Investigar el desove y desarrollo intracapsular y larvario de esta especie como herramientas para su producción en cautiverio con fines de repoblamiento. Métodos: Se recolectaron 18 progenitores que fueron mantenidos en un sistema cerrado con parámetros y alimentación controladas durante abril 2017 hasta septiembre 2018. Se registró: crecimiento de progenitores, desove de masas ovígeras, cantidad de cápsulas y número de embriones y larvas dentro de las cápsulas. La temperatura de incubación fue de $28.2 \pm 1.5{ }^{\circ} \mathrm{C}$. Las principales estructuras morfológicas de embriones y larvas fueron documentadas conforme su desarrollo. Resultados: El desarrollo embrionario intracapsular y de la larva velígera hasta su asentamiento tuvieron una duración de 14-15 y 31-32 días, respectivamente. Cada hembra de 80-88 $\mathrm{mm}$ de longitud produjo alrededor de 4 masas ovígeras, 627 cápsulas y 663993 larvas velígeras extracapsulares por año. Conclusiones: La fácil adaptación y el potencial reproductivo mostrado por $H$. nigritus en laboratorio se presentan como estrategias promisorias para su repoblación y conservación.

Palabras clave: condiciones controladas; conservación; desarrollo embrionario; estrategia reproductiva; gastrópodos; Golfo de California.

\section{REFERENCIAS}

Barnes, R.S.K. (1990). Reproductive strategies in contrasting populations of the coastal gastropod Hydrobia ulvae. II. Longevity and life-time egg production. Journal of Experimental Marine Biology and Ecology, 138(3), 183-200. DOI: 10.1016/0022-0981(90)90166-A

Bayne, B.L. (1976). Aspects of reproduction in bivalve molluscs. Estuarine Processes. Uses, Stresses, and Adaptation to the Estuary. Massachusetts, United States of America: Academic Press.

Bayne, B.L., \& Newell, R.C. (1983). 9. Physiological energetics of marine molluscs. The Mollusca. 
Physiology. Massachusetts, United States of America: Academic Press.

Benkendorff, K., Bremner, J.B., \& Davis, A.R. (2000). Tyrian purple precursors in the egg masses of the Australian muricid, Dicathais orbita: A possible defensive role. Journal of Chemical Ecology, 26(4), 1037-1050. DOI: 10.1023/A:1005441116095

Benkendorff, K., Westley, C.B., \& Gallardo, C.S. (2004). Observations on the production of purple pigments in the egg capsules, hypobranchial and reproductive glands from seven species of Muricidae (Gastropoda: Mollusca). Invertebrate Reproduction \& Development, 46(2-3): 93-102. DOI: $10.1080 / 07924259.2004 .9652612$

Brokordt, K. (2003). Sex differences in reproductive investment: maternal care reduces escape response capacity in the whelk Buccinum undatum. Journal of Experimental Marine Biology and Ecology, 291, 161180. DOI: 10.1016/S0022-0981(03)00119-9

Brusca, R.C., Kimrey, E., \& Moore, W. (2004). A seashore guide to the northern Gulf of California. Tucson, United States of America: Arizona-Sonora Desert Museum.

Buckland-Nicks, J., \& Chia, F.S. (1990). Egg capsule formation and hatching in the marine snail Littorina sitkana. Philosophical Transactions of the Royal Society of London B, 326, 159-176.

Camacho-Sánchez, F.Y. (2009). Efecto de la temperatura sobre el desarrollo embrionario del caracol burro Hexaplex (Muricanthus) nigritus (Philippi, 1845), en condiciones de laboratorio (Tesis de Licenciatura). Universidad de Occidente, Sinaloa, México.

Camacho-Sánchez, F.Y. (2012). Desarrollo embrionario $y$ metamorfosis del caracol chino Hexaplex (Muricanthus) nigritus (Philipi, 1845) en condiciones de laboratorio (Tesis de Maestría). Instituto Politécnico Nacional, Centro Interdisciplinario de Investigación para el Desarrollo Integral Regional, México.

Cañete, J.I., Gallardo, C.S., Céspedes, T., Cárdenas, C.A., \& Santana, M. (2012). Encapsulated development, spawning and early veliger of the ranellid snail Fusitriton magellanicus (Röding, 1798) in the cold waters of the Magellan Strait, Chile. Latin American Journal of Aquatic Research, 40(4), 914-928. DOI: 103856/ vol40-issue4-fulltext-8

Chaparro, O.R., \& Flores, M.L. (2002). Reproductive output of Crepidula fecunda females: distribution of energy in the production of gametes and capsules walls. New Zealand Journal of Marine and Freshwater Research, 36, 661-673. DOI: 10.1080/00288330.2002.9517122

Chatzinikolaou, E., \& Richardson, C.A. (2010). Parental size and environmental conditions affect egg capsule production by Nassarius reticulatus (Linnaeus 1758) (Gastropoda: Nassariidae). Journal of
Experimental Marine Biology and Ecology, 390, 14-21. DOI: 10.1016/j.jembe.2010.04.029

CITES. (2020). Convención sobre el Comercio Internacional de Especies Amenazadas de Fauna y Flora Silvestres. Recuperado de www.ckecklist.cites.org/\#/es

CONAPESCA. (2020). Comisión Nacional de Acuacultura y Pesca. Recuperado de www.acuasesor.conapesca. gob.mx/anuario.php

Cudney-Bueno, R. (2007). Marine reserves, communitybased management, and small-scale benthic fisheries in the Gulf of California, Mexico (Doctoral Dissertartion). University of Arizona, Arizona, United States of America.

Cudney-Bueno, R., \& Rowell, K. (2008). The black murex snail, Hexaplex nigritus (Mollusca, Muricidae), in the Gulf of California, Mexico: II. Growth, longevity, and morphological variations with implications for management of a rapidly declining fishery. Bulletin of Marine Science, 83(2), 299-313.

Cudney-Bueno, R., Prescott, R., \& Hinojosa-Huerta, O. (2008). The black murex snail, Hexaplex nigritus (Mollusca, Muricidae), in the Gulf of California, Mexico: I. Reproductive ecology and breeding aggregations. Bulletin of Marine Science, 83(2), 285-298.

Cumplido, M., Pappalardo, P., Fernández, M., Averjub, A., \& Bigatti, G. (2011). Embryonic develoment, feeding and intracapsular availability in Thophon geversianus (Gastropods: Muricidae). Journal of Molluscan Studies, 77, 429-436. DOI: 10.1093/mollus/eyr025

Dame, R.F. (1996). Ecology of marine bivalves: an ecosystem approach. Boca Raton, United States of America: CRC Press.

D’Asaro, C.N. (1966). The egg capsules, embryogenesis, and early organogenesis of a common oyster predator, Thais haemastoma floridana (Gastropoda: Prosobranchia). Bulletin of Marine Sciences, 16(4), 884-914.

D’Asaro, C.N. (1970). Egg capsules of prosobranch mollusks from South Florida and The Bahamas and notes on spawning in the laboratory. Bulletin of Marine Sciences, 20(2), 414-440.

D'Asaro, C.N. (1992). Gunnar Thorson's world-wide collection of prosobranch egg capsules: Muricidae. Ophelia, 35(1), 1-101.

Fabioux, C., Huvet, A., Le Souchu, P., Le Pennec, M., \& Pouvreau, S. (2005). Temperature and photoperiod drive Crassostrea gigas reproductive internal clock. Aquaculture, 250(1-2), 458-470. DOI: 10.1016/j. aquaculture.2005.02.038

Fu, T., Guerette, P.A., Tan, R.Y.T., Zhao, H., Schefer, L., Mezzenga, R., \& Miserez, A. (2015). Biomimetic self-assambly of recombinant marine snail egg capsule proteins into structural coiled-coil units. 
Journal of Materials Chemistry B, 3, 2671-2684. DOI: $10.1039 / \mathrm{c} 4 \mathrm{tb} 01434 \mathrm{k}$

Góngora, G.A. (1999). Efecto de la temperatura sobre el desarrollo embrionario del caracol marino Strombus pugilis (L.) (Tesis de Licenciatura). Universidad Autónoma de Yucatán, Yucatán, México.

Góngora-Gómez, A.M., Domínguez-Orozco, A.L., Muñoz-Sevilla, N.P., \& Rodríguez-Gil, L.A. (2007). Obtención de masas ovígeras del caracol lancetilla, Strombus pugilis (Mesogastropoda: Strombidae) en condiciones de laboratorio. Revista de Biología Tropical, 55(1), 183-188.

Góngora-Gómez, A.M., García-Ulloa, M., \& Domínguez, A. (2010). Desarrollo embrionario del caracol chino Muricanthus nigritus para su repoblación y preservación en Guasave, Sinaloa, México. In J.C. ChávezComparán \& J. Mimbela-López (Eds.), Avances sobre Investigaciones Marinas y Acuicolas del Pacifico Mexicano ( $1^{\mathrm{a}}$ ed., pp 112-117). Colima, México: Universidad de Colima.

Góngora-Gómez, A.M., García-Ulloa, M., DomínguezOrozco, A.L., \& Camacho-Sánchez, F.Y. (2011). Aspectos reproductivos cuantitativos del caracol murex negro, Hexaplex nigritus (Philipi, 1845) en condiciones de laboratorio. Ciencia y Mar, $X V(44), 31-34$

González, K.A., \& Gallardo, C.S. (1999). Embryonic and larval development of the muricid snail Chorus giganteus (Lesson, 1829) with and assessment of the developmental nutrition source. Ophelia, 51(2), 77-92. DOI: 10.1080/00785326.1999.10409400

Güler, M., \& Lök, A. (2014). Embryonic development and intracapsular feeding in Hexaplex trunculus (Gastropoda: Muricidae). Marine Ecology, 35, 193-203. DOI: $10.1111 /$ maec.12066

Ito, K. (1997). Egg-size and -number variations related to maternal size and egg, and the relationship between egg size and larval characteristics in an annual marine gastropod, Haloa japonica (Opisthobranchia; Cephalaspidea). Marine Ecology Progress Series, 152, 187-195.

Jagadis, I., Shunmugasundaram, K., Sathakkathulla, Md., \& Mohanraj, T. (2013). Spawning, intracapsular development and production potential of viable juveniles of a murex Chicoreus virgineus var. ponderosa Sowerby under laboratory conditions. Agricultural Sciences, 4, 244-248. DOI: 10.4236/as.2013.45035

Jagadis, I., Kavitha, M., Padmanathan, J., Maharshi, A., \& Varadarajakumar, A. (2017). Lessons on broodstock maintenance, spawning, larval rearing and juvenile production of marine gastropods of ornamental value. Aquaculture Research, 48(5), 2581-2592. DOI: 10.1111/are. 13094
Lima, G., \& Pechenik, J. (1985). The influence of temperature on growth rate and length of larval life of the gastropod, Crepidula plana Say. Journal of Experimental Marine Biology and Ecology, 90, 55-71. DOI: 10.1016/0022-0981(85)90074-7

Lord, A. (1986). Are the contents of egg capsules of the marine gastropod Nucella lapillus (L.) axenic? American Malacological Bulletin, 42, 201-203.

Mahmoud, M.A.M., Mohammed, T.A.A., \& Yassien, M.H. (2013). Spawning frequency, larval development and growth of Muricid gastropod Chicoreus ramosus (Linnaeus, 1758) in the Laboratory at Hurghada, Northern red Sea, Egypt. Egyptian Journal of Aquatic Research, 39, 125-131. DOI: 10.1016/j. ejar.2013.07.002

Moffa, R., Chávez, J., Bastardo, G., Villalba, W., \& Crescini, R. (2017). Desarrollo intracapsular del gasterópodo Fasciolaria tulipa (Linnaeus, 1758) (Mollusca: Neograstropoda: Fasciolariidae) en condiciones de laboratorio. Amici Molluscarum, 25(1-2), 19-26.

Munguía-Vega, A., Torre, J., Turk-Boyer, P., Marinone, G., Lavin, M., ... Cudney-Bueno, R. (2015). PANGAS: An Interdisciplinary Ecosystem-Based Research Framework for Small-Scale Fisheries in the Northern Gulf of California. Journal of the Southwest, 57(2-3), 337-390.

Naegel, L., \& Cooksey, C.J. (2002). Tyrian purple from marine muricids, especially from Plicopurpura pansa (Gould, 1853). Journal of Shellfish Research, 21(1), 193-200.

Naegel, L.C.A., \& Gómez del Prado-Rosas, M.C. (2004). Embriogénesis y desarrollo larvario intra-capsular de Plicopurpura pansa (Gould, 1853) (Prosobranchia, Muricidae) en condiciones de laboratorio. Ciencias Marinas, 30(2), 297-310.

Najmudeen, T.M., \& Victor, A.C.C. (2004). Seed production and juvenile rearing of the tropical abalone Haliotis varia Linnaeus 1758. Aquaculture, 234, $277-$ 292. DOI: $10.1016 /$ j.aquaculture.2003.12.013

Navarro, J.M., Leiva, G.E., Gallardo, C.S., \& Varela, C. (2002). Influence of diet and temperature on physiological energetics of Chorus giganteus (Gastropoda: Muricidae) during reproduction conditioning. New Zealand Journal of Marine and Freshwater Research, 36, 321-332. DOI: 10.1080/00288330.2002.9517089

Pastorino, G., Penchaszadeh, P.E., \& Scarabino, F. (2007). Egg capsules, eggs and embryos of the southwestern Atlantic gastropod Coronium coronatum (Gastropods: Muricidae). Journal of Molluscan Studies, 73, 61-65. DOI: 10.1093/mollus/eyp024

Pechenik, J., \& Lima, G. (1984). Relationship between growth, differentiation, and length of larval life for individually reared larvae of the marine 
gastropod, Crepidula fornicata. The Biological Bulletin, 166, 537-54.

Pechenik, J.A., Chang, S.C., \& Lord, A. (1984). Encapsulated development of the marine prosobranch gastropod Nucella lapillus. Marine Biology, 78, 223-229. DOI: $10.1007 / \mathrm{BF} 00394705$

Poutiers, J.M. (1995). Bivalvos. In W. Fischer, F. Krupp, W. Schneider, C. Sommer, K. Carpenter, \& V.H. Neim (Eds.), Guía FAO para la identificación de especies para los fines de pesca: Pacífico CentroOriental: Invertebrados ( $1^{\text {st. }}$ ed., pp. 224-297). Rome, Italy: Food and Agriculture Organization.

Prescott, R., \& Cudney-Bueno, R. (2008). Mobile 'reefs' in the northeastern Gulf of California: aggregations of black murex snails Hexaplex nigritus as habitat for invertebrates. Marine Ecology Progress Series, 367, 185-192. DOI: 10.3354/meps07450

R Core Team. (2018). R: A language and environment for statistical computing. R Foundation for Statistical Computing, Vienna, Austria. Retrieved from: https:// www.R-project.org

Rawlings, T.A. (1995). Direct observation of encapsulated development in Muricid Gastropods. The Veliger, $38(1), 54-60$.

Riquelme, C.E., \& Chávez, P.C. (1991). Colonization of vibrios on developmental stages of Concholepas concholepas (Bruguière, 1789) (Mollusca: Muricidae). In K. Koop (Ed.), Ecology of Marine Aquaculture. Workshop on Research in Aquaculture (1 ${ }^{\text {st }}$. ed., pp. 84-95). Chile: International Foundation for Science.

Romero, M.S., Lohrmann, K., Bellolio, G., \& Dupre, E. (2003). Comparative observations on reproduction, spawning, and early veligers of three common subtidal mesograstropods from North Central Chile. The Veliger, 46(1), 50-59.

Romero, M.S., Gallardo, C.S., \& Bellolio, G. (2004). Egg laying and embryonic-larval development in the snail Thais (Stramontia) chocolata (Duclos, 1832) with observations on its evolutionary relationships within the Muricidae. Marine Biology, 145(4), 681-692. DOI 10.1007/s00227-004-1368-9

Schwab, D.B., \& Allen, J.D. (2014). Size-specific maternal effects in response to predator cues in an intertidal snail. Marine Ecology Progress Series, 499, 127-141. DOI: $10.3354 /$ meps 10652

SEMARNAT. (2020). Secretaría de Medio Ambiente y Recursos Naturales. Recuperado de www.dgeiawf. semarnat.gob.mx:8080/ibi apps/WFServlet?IBIF. ex $=$ D2_PESCA01_03 \&IBIC_user=dgeia. mce \&IBIC_pass $=$ dgeia_mce $\& N O M B R E A N I O=*$

Smith, K.E., \& Thatje, S. (2013). Nurse egg consumption and intracapsular development in the common whelk Buccinum undatum (Linnaeus 1758). Helgoland Marine Research, 67, 109-120. DOI: 10.1007/ s10152-012-0308-1

Stump, T.E. (1975). Pleistocene molluscan paleoecology and community structure of the Puerto Libertad region, Sonora, Mexico. Palaeogeography, Palaeoclimatology, Palaeoecology, 17(3), 177-226.

Wayne, N.L. (2001). Regulation of seasonal reproduction in mollusks. Journal of Biological Rhythms, 16(4), 391-402. DOI: 10.1177/074873001129002097

Zupo, V., \& Patti, F.P. (2009). Laboratory spawning, larval development and metamorphosis of the marine snail Nassarius reticulatus (L.) (Caenogastropoda, Nassaridae). Invertebrate Reproduction and Development, 53(1), 23-31. DOI: 10.1080/07924259.2009.9652286 